\title{
La formazione di Giovanni Battista Antonelli: note storiche e contesto sociale prima del suo arrivo in Spagna
}

\section{Gianluca Useli', Maurizio d'Amato ${ }^{\text {b }}$}

${ }^{\mathrm{a}}$ Architect, Firenze, Italy, uselig@libero.it ${ }^{\mathrm{b}}$ Professor Architect, Firenze, Italy, maurizio.d.amatofirenze@email.ita,

\begin{abstract}
Giovanni Battista Antonelli before his arrival in Spain in 1559, being in his thirties, had spent the first part of his life in his native country, Italy. The present research concentrates on this territory and those years: a context of wars, captains, architects, theorists and treatises, many known and less known personalities who influenced Antonelli military engineering education.

More detailed information appear in Italian archives about Giovan Francesco Guidi di Bagno and Antonelli: the first, Count of Montebello, was an important mercenary captain for the more powerful personalities, while the second was, at that time, at his service. Fiduciary and family relationships interlinked around the Count Guidi and others eminent personalities such as Giovanni Battista Belluzzi called "Sanmarino", Cosimo I Medici up to Juan Manrique de Lara and Vespasiano Gonzaga Colonna, to mention the main ones.

This investigation tries to shed light on the first part of Antonelli learning, as he took part to the War between Florence and Siena and in the Naples Kingdom. He subsequently left for Flanders with the Spanish army and reaching eventually Spain. During Felipe II Reign, after having written the Epitomi, he started the well known career of Antonelli family as military engineers. It was especially Battista, the younger brother, one of the main representatives in the history of military engineering, especially between the 16 th and 17 th centuries.
\end{abstract}

Keywords: Antonelli, education, biographies, Italian archives, 16th century.

\section{Introduzione}

Tre generazioni di ingegneri militari si avvicendarono all'interno della famiglia Antonelli, attive a partire dalla metà XVI secolo su buona parte dell'impero spagnolo che si estendeva dal fronte mediterraneo fino a quello atlantico. Gli studi condotti fino ad oggi hanno permesso di delineare gran parte dell'operato di questi ingegneri di origine italiana; la ricostruzione è stata possibile grazie all'enorme quantità di documenti conservati negli Archivi Spagnoli e costituiti da un corposo carteggio di comunicazioni e disegni, alcuni testamenti ed il manoscritto delle Epitomi. Occorre sottolineare invece, la scarsità di notizie relative al periodo precedente l'arrivo in terra spagnola della prima generazione, costituita dai fratelli Giovanni Battista e Battista Antonelli, nativi di Gatteo. I due avevano maturato una tale esperienza in Italia che permise loro di soddisfare i primi incarichi di una certa levatura a partire dal 1561 a fianco dell'umanista e condottiero Vespasiano Gonzaga Colonna, Viceré del Regno di Valencia su nomina di Filippo II.

Il fratello maggiore Giovanni Battista (15271588) compie il suo arrivo in Spagna intorno al 1559 , unito alle truppe spagnole di rientro dalle campagne militari nelle Fiandre. Invece Battista (1543/5-1616), forse su invito del 
fratello, approderà in territorio spagnolo tra il 1570 e il 1571, proveniente dall'assedio di Famagosta ad opera dei Turchi contro la Repubblica di Venezia. Questa costituisce per adesso l'unica informazione al riguardo di Battista Antonelli, dunque troppo poco per argomentare circa la sua formazione in Italia. Per quanto riguarda Giovanni Battista vi sono innanzitutto le Epitomi redatte tra il 1560 ed il 1561, che in primo luogo testimoniano le sue conoscenze fino a quel momento, ed in secondo luogo forniscono ulteriori informazioni riguardanti il periodo poco precedente: il coinvolgimento nel 1557 nelle battaglie di San Quintino e di Dourlens nelle Fiandre.

A completamento delle fonti riguardanti il periodo di studio, un manoscritto settecentesco, conservato alla Biblioteca Malatestiana di Cesena, ci fornisce un paio di informazioni interessanti: la professione del padre Girolamo, capomastro con una bottega edile a Gatteo e la partecipazione di Giovanni Battista alla battaglia di Siena (1553-55) sotto le fila del capitano di ventura al servizio di Cosimo I de' Medici, il conte Giovan Francesco Guidi da Bagno. In quella occasione l'Antonelli, nelle vesti di "segretario et cancelliere della compagnia di lancie et di archibugieri a cavallo" del Guidi, si rese protagonista nel 1554 del trafugamento dei resti del Beato Colombini dalla chiesa di Santa Bonda nei pressi di Siena. Queste fonti rappresentano il punto di partenza dello studio, nel tentativo di interpretare i contributi alla sua formazione.

\section{La formazione dell'ingegnere militare tra teoria e pratica}

Nel XVI secolo si andava sempre più delineando il nuovo ruolo dell'ingegnere $o$ architetto militare, dotato di profonde conoscenze tecniche, scientifiche e strategiche. La tecnologia delle armi avanzava con l'invenzione di nuove artiglierie, di conseguenza mutavano le strategie di offesa e difesa, così le forme e le concezioni delle fortezze e dei ripari. In generale il campo delle armi si complicava, chiamando in gioco varie discipline scientifiche. L'ingegnere militare doveva essere conoscitore del nuovo modo di fare poliorcetica, del cambiamento del modo di operare militarmente: si lasciava alle spalle una guerra costituita esclusivamente da cavalieri e scudieri, ed ora basata principalmente sull'abbattimento di difese tramite pesanti ordigni. La nuova artiglieria mutava infatti il tipo di scontro, non più sul corpo a corpo ma bensì a distanza, giocato su rapporti di costobenefici e ricompense finali e solo successivamente di razzia e depredazione della popolazione sconfitta e inerme.

L'ingegnere militare era dunque una figura senz'altro tecnica ma anche d'intellettualestratega. La sua formazione presupponeva, in tempo di pace:

a) conoscenze nel campo della geometria e delle scienze matematiche, fisiche e chimiche,

b) saper progettare, coordinare e dirigere le opere difensive sia in muratura che in terra,

c) avere nozioni tecniche di cantiere approfondite ed esperienza di conduzione di esso,

d) aver partecipato, nelle retrovie di appoggio, ad eventuali campagne militari,

e) capacità di studio dei territori, per la scelta dei siti, percorsi e strategie di offesa e difesa,

f) saper leggere, possibilmente anche in latino, per l'accesso a eventuali testi pubblicati o manoscritti,

g) avere nozioni approfondite d'idraulica,

h) essere esperto di artiglierie e delle relative gittate (balistica),

i) conoscere la siderurgia e i processi di fusione,

j) avere nozioni pirotecniche per la fabbricazione delle polveri ottenute dal salnitro,

k) saper coordinare i lavori di carpenteria per la realizzazione di eventuali macchine da guerra o argani importanti,

1) capacità di amministrare e rendicontare tutto ciò che riguardi la progettazione, la costruzione della fortezza, che doveva essere 
occupata da una guarnigione minima, ma ottimale per la sua difesa ed il mantenimento a minori spese.

In tempo di guerra, invece, l'ingegnere doveva mettere in atto:

a) la sua capacità di valutare tecnicamente le caratteristiche della fortezza nemica con i suoi lati architettonici più deboli, sia "di dentro" che "di fuori", al fine di appoggiare le strategie di attacco,

b) le sue conoscenze e capacità di lettura dei siti con lo scopo di condizionare fonti di approvvigionamento e vettovagliamento durante un assedio,

c) il bagaglio tecnico per poter consigliare, scegliere e costruire un accampamento sicuro e salubre,

d) il saper costruire macchine da guerra adattando le esigenze belliche alla disponibilità in loco delle materie prime,

e) la realizzazione di opere provvisionali per varcare con l'artiglieria corsi d'acqua,

f) in caso di danneggiamento dell'opera difensiva, il ripristino e l'adattamento successivo,

g) eventuali valutazioni dei limiti e degli interventi da potere riutilizzare nella progettazione di opere successive,

h) la funzione di figura propositiva $\mathrm{e}$ contemporaneamente esecutiva del Capitano Generale, offrendogli risposte ed indicazioni tecniche e operative precise,

i) la valutazione delle spese al fine di essere dimostrabili a operazione militare conclusa.

Per chiarire le difficoltà nelle quali si dibatteva l'ingegnere militare, bisogna però aver presente il contesto scientifico di quel tempo: ad esempio la chimica, per la lavorazione del salnitro, e la siderurgia erano basate ancora su processi alchemici; oppure la gittata di un pezzo d'artiglieria era fondata sulla sperimentazione, e pur avendo individuato l'importanza dell'alzata, del rapporto fra quantità, qualità di polvere e del peso delle munizioni, non se ne era ancora intuito l'aspetto analitico.
Negli stessi anni l'industria tipografica, la veneziana in particolare, intraprese una produzione libraria incredibile per quantità e vastità d'argomenti trattati. Nello specifico, i testi ad argomento militare erano sorprendentemente numerosi. Al ruolo dell'ingegnere ed al cambiamento delle strategie di guerra dunque, si aggiunse una decisa veicolazione delle novità, tramite la stampa ed i manoscritti di uso personale, che andavano letteralmente a ruba. I motivi d'interesse per la loro redazione erano i più svariati: potevano essere stesi per esplicita richiesta di un'alta carica politica e militare, oppure semplici ricordanze delle proprie conoscenze, nella speranza di poter ricevere un patrocinio editoriale oppure un incarico lavorativo. L'acquisizione di un testo poteva avvenire per via commerciale, sottrazione in tempo di guerra, omaggio ricevuto in copia o smarrimento.

Nel ricercare quindi le nozioni originali ivi contenute, occorre risalire alla data del manoscritto autografo, o ristretto o copia fraudolenta, piuttosto che fare riferimento alla data di stampa poiché spesso postuma, mentre i contenuti erano già in possesso agli addetti ai lavori. Inoltre a Venezia, l'avallo o il nulla osta per la pubblicazione era disciplinato dalla Chiesa, in un procedimento molto lento e imperscrutabile: il manoscritto giaceva incustodito per molto tempo e quando arrivava il parere, questo poteva essere negativo, per cui bisognava ricorrere a storpiature del nome dell'autore o trovare un uomo di paglia, come era solito fare il Meietti, inondando l'Europa di testi a stampa "proibiti".

\section{Le Epitomi}

Le tre epitomi di Giovanni Battista Antonelli sono state scritte a Toledo tra il 1560 ed il 1561. La loro pubblicazione anastatica del 2007 a cura di Sartor ha reso pubblico questo prezioso documento. Sono raccolte in un unico volume, attualmente conservato al Museo del Ejército di Toledo, che è così composto:

1) Epitomi delle fortificationi moderne (datate 1560) 
2) Epitomi del Trattato dell'Artiglieria (datate marzo 1561)

3) Epitomi della manera di alloggiare un campo (datate 24 aprile 1560)

Nella recente pubblicazione, Sartor e La Torre Echavarri affrontano in modo sistematico il manoscritto dell'Antonelli, con nutriti commenti e riflessioni. In tale occasione preme sottolineare solo alcuni aspetti: Antonelli aveva scritto già un trattatello che affermava essere andato perduto, nonché si prometteva di scriverne altri che probabilmente avrebbero reso più corposa la prima delle epitomi, circa le fortificazioni, intento poi disatteso; l'altro riguarda le dediche che egli include nei manoscritti: le prime due, per ordine di tempo di scrittura, ossia quella sull'alloggiamento del campo e quindi quella sulle fortificazioni sono dedicate a Juan Manrique de Lara, Clavero di Calatrava e Capitano Generale dell'Artiglieria durante la campagna nelle Fiandre, mentre l'ultima sull'artiglieria è stata dedicata direttamente al sovrano Filippo II, al quale si rivolge in tono non troppo formale lasciando intendere che vi fosse già stato un contatto diretto. Altro dato che risalta è che le epitomi furono espressamente scritte su richiesta di Manrique de Lara. Da alcuni passi si evince che il Capitano Generale desiderasse dei trattati ad argomento militare che fossero "non prolissamente scritti", e così l'Antonelli fece. Infatti il termine epitome vale quale "sunto sostanziale d'un'opera specialmente storica", dunque già nel titolo palesa che non nasce come opera originale ma bensì parafrasi e riassunto di opere già esistenti. Sicuramente l'ingegnere apprese alcune conoscenze anche sui campi di battaglia, ben individuabili nelle epitomi dell'alloggiamento ed in alcuni passi di quelle dell'artiglieria. Per quest'ultime, l'Antonelli stesso, nella prefazione dichiara di mettere per iscritto anche le conoscenze che il capitano Manrique possedeva e gli condivise a San Quintino.

Nell'intenzione di risalire dunque all'originalità degli autori nella trattatistica di allora è compito arduo, poiché i trattatisti, oltre ad essere numerosi, riportavano conoscenze spesso simili $\mathrm{o}$ addirittura copiate. Il confronto sistematico tra i passi delle Epitomi con quelli del Lanteri come del Cattaneo, o Zanchi, De Marchi, Leonardi per tornare indietro nel tempo con i classici Vitruvio e Vegezio ed i più recenti Alberti, Di Giorgio Martini, e Tartaglia, può risultare infine dispersivo. Sicuramente Antonelli avrà letto e studiato gli scritti di tanti autori di riferimento, ma le analogie con almeno le opere di due di questi influenti personaggi dell'epoca è piuttosto eloquente: Giovanni Battista Belluzzi "Sanmarino" e Vannuccio Biringuccio o Biringucci.

Il conoscimento di Giovanni Battista Belluzzi di San Marino (1506-1554) ebbe una profonda influenza sulla formazione di Giovanni Battista Antonelli. Tale affermazione è risultato della ricostruzione della vita biografica e professionale del primo, che presenta continue connessioni con il secondo, sorrette dalle evidenti analogie di contenuti tra il manoscritto originale del Belluzzi e le Epitomi, che l'Antonelli confermerà ancora nei rapporti inviati alla Corte durante le spedizioni nel Levante spagnolo.

Per inquadrare l'ingegnere di San Marino ci viene in aiuto il prezioso studio monografico della Lamberini (2007), dal quale emergono diversi documenti autografi importanti. Dal diario biografico, scritto dal 1535 al 1541, si evince in modo chiaro quale fu a sua volta la formazione del Belluzzi. Sinceramente fedele alla patria natia, la Repubblica di San Marino, ebbe comunque la fortuna di viaggiare, stare a contatto con tantissimi tra i più potenti dell'epoca, legandosi a loro con rapporti sia familiari quanto professionali. Se ne evidenziano alcuni passaggi: un primo breve periodo a Roma alle dipendenze di Ascanio Colonna, seguito dal sodalizio con il suocero Girolamo Genga alla corte di Francesco Maria I della Rovere, fino alla nomina di primo ingegnere di Cosimo I Medici ed il coinvolgimento alla guerra di Siena dove perse la vita nel 1554. Proprio durante il periodo alla corte medicea redige due manoscritti, il primo sulle fortificazioni di terra concluso nel 1545 e il secondo sulle fortificazioni in generale, consegnato nel 1554 , prima della sua morte, al suo allievo Bernardo 
Puccini. In realtà va detto che il secondo manoscritto non è altro che la prosecuzione del primo, il quale ricordiamo fu consegnato in copia nel 1545 a Chiappino Vitelli. Tale ristretto, dove il Belluzzi promuoveva la tecnica del terrapieno e delle rapide ed efficienti opere in terra argillosa, resistenti alle nuove artiglierie, ebbe grande diffusione e se ne produsse numerose copie.

Una di queste copie dovrebbe essere passata per le mani dell'Antonelli, il quale, nella prima Epitome sulle fortificazioni, ripropone in modo sostanziale. Se ne riconosce la scaletta adottata, la terminologia, le parole chiave e i vari corollari, le tecniche di realizzazione e concatenazione degli argomenti, una volta sfrondati di ogni valore letterario o retorico. Dunque l'Antonelli, alla pari di altri, sebbene non ci è noto quale fu il tramite diretto, fu abile recettore del codice belluzziano e correttamente lo fece proprio sia nella scrittura delle Epitomi, sia nel suo operato in Spagna.

La seconda Epitome, composta da tre libri, risulta il trattato più corposo e articolato. Anche in questo caso l'Antonelli, per la sua stesura, si è basato sui contenuti di un altro testo: De la Pirotechnia del senese Biringuccio pubblicato postumo nel 1540 . L'ingegnere romagnolo ne ripete i principi con modalità già descritti e adottati nella prima epitome. In questo caso però, adatta il testo originale ad un altro filo logico, quindi mescola gli argomenti precedentemente trattati dal senese, per recuperarli secondo il suo schema; le varie titolazioni rinviano comunque al testo già pubblicato, conservandone le parole caratterizzanti, che potremmo definire parole-spia.

Il testo della terza Epitome, più breve degli altri, è principalmente corredato da un ampio campione di disegni che descrivono alloggiamenti con vari tipi di distribuzioni interne in base al numero di lati sicuri naturali. Trattasi in questo caso del risultato di letture di autori classici, come era consuetudine sull'argomento, unito all'esperienza maturata nel ruolo di aiutante del maestro di campo Jean de
Ligne Conte di Aremberg durante la campagna di Dourlens nelle Fiandre.

\section{L'Antonelli nel campo di battaglia}

Le fonti ci documentano la presenza di Giovan Battista alle seguenti campagne militari, schierato nel fronte spagnolo: la guerra di Siena condotta da Cosimo I (tra il 1553 ed il 1555) e le battaglie delle Fiandre di San Quintino e Dourlens (tra il 1556 ed il 1557). Il filo conduttore della ricerca è rappresentato da due personaggi che sappiamo essere entrati in contatto con l'Antonelli: il Conte Giovanni Francesco Guidi e Il Capitano Juan Manrique de Lara.

Il conte Guidi di Bagno, nacque nei primi anni del secolo a Cesena, iniziando la sua carriera militare al soldo dello Stato Pontificio. Nel 1549 il Conte riceve dal Papa l'elezione a Marchese di Montebello, includendo Gatteo tra i suoi feudi, e viene dotato di un corposo esercito di fanti e cavalli. Il Guidi si renderà protagonista nel 1551 nell'assedio della Mirandola, che vedeva contrapposti l'esercito imperiale e pontificio contro $\mathrm{i}$ francesi in appoggio al Ducato di Parma. Tralasciando i dettagli, l'evento risultò celebre poiché il piccolo borgo della Mirandola, grazie alla sua fortificazione di moderno concetto e dotata delle nuove artiglierie, dimostrò ottima capacità difensiva contro un esercito oltre dieci volte più numeroso. L'ipotesi che l'Antonelli abbia partecipato a tale campagna, forse proprio al fianco del Guidi, non è documentato ma possiamo ipotizzarlo per vari motivi. Il Conte Guidi, come detto, era stato appena dotato di un importante esercito al suo seguito, dunque dovette assoldare diversi uomini per gestirlo. Infine l'allusione è supportata da una dichiarazione dello stesso Antonelli del 1575 con la quale affermava di servire la Spagna da 24 anni, dunque proprio dal 1551 .

A questo punto il passaggio successivo ci porta a Siena, dove il dato è ora più documentabile: Antonelli partecipò alla guerra e vi era giunto con la compagnia del Conte Guidi. Lo scontro tra Firenze e Siena vedeva coinvolto Cosimo I, che godeva dell'appoggio imperiale e spagnolo, contro il più noto degli esiliati fiorentini, il 
condottiero Piero Strozzi, schierato dalla parte dell'esercito francese. La guerra tra le parti fu complessa e lunga, tra il 1553 ed il 1555, finanziata direttamente dall'imperatore Carlo V. Anche stavolta lo scontro rappresentava un importante crocevia dal punto di vista strategico militare e della messa in opera dei nuovi dettami della poliorcetica. La quantità d'ingegneri coinvolti nel conflitto con Siena è numerosa, uno su tutti Giovanni Battista Belluzzi, il quale oltre a ricoprire un ruolo significativo nelle operazioni militari, durante le stesse perse la vita. Le connessioni tra il Belluzzi ed il Guidi in realtà, risalivano già a qualche anno prima. Il Belluzzi, fin da giovane, era solito frequentare i luoghi dello stesso Conte Guidi, in particolare al castello di Montebello. Come egli stesso racconta nel suo diario autobiografico, a Montebello dimorava il castellano Arcangelo, divenuto suo cognato per averne sposato la sorella Diana. Il Belluzzi annotava con continuità le vicende riguardanti Montebello, Arcangelo, gli affari di famiglia e conseguentemente del Guidi. Il diario in realtà si interrompe nel 1541, data per la quale è difficile anche solo ipotizzare che un Antonelli quattordicenne fosse già alle dipendenze del Guidi. Tuttavia non è da escludere che la conoscenza tra Antonelli e Belluzzi fosse realmente avvenuta qualche anno dopo, comunque prima del 1554.

Tornando alla guerra di Siena, va segnalato che nell'esercito spagnolo invece figuravano due importanti personaggi chiave per gli anni a seguire dell'Antonelli: un giovane Filippo II (1527-1598), futuro sovrano che si formava alle armi al fianco del Duca d'Alba (forse seguito anche dal paggio di fiducia Vespasiano Gonzaga) ed il Capitano Generale Juan Manrique de Lara (legato al Regno di Napoli). È probabile dunque che, durante lo scontro, il Conte Guidi fosse entrato a stretto contatto con l'esercito spagnolo, per via probabilmente anche del legame con lo stato pontificio. A conferma del coinvolgimento "spagnolo" del Guidi di quegli anni ci giunge un documento conservato all'Archivio storico di Montebello. La carta consiste in una patente datata 10 giugno 1553 in Viterbo a firma di Manrique de Lara, con la quale attribuiva l'investitura del Conte Guidi di Bagno a capo di una compagnia di mille fanti a servizio dell'Imperatore con incarico ricevuto dal cardinale di Jaen, Pedro Pacheco (nonché Viceré di Napoli). In modo indiretto siamo in possesso dunque del primo anello che lega l'Antonelli a Manrique de Lara, e che lo porterà di lì a poco a seguirlo nelle Fiandre. La guerra di Siena infatti terminerà nel 1555, un anno dopo la morte del Belluzzi e dopo che Guidi e Antonelli avevano fatto rientro in Romagna nel 1554. È probabile che da quella occasione, o poco dopo, i due si dividono poiché il Guidi si rende protagonista di un fatto di cronaca che lo screditerà dinnanzi al pontefice, costringendolo $\mathrm{a}$ fughe ed alla perdita dei suoi feudi. L'Antonelli invece, dopo aver portato le reliquie del Beato Angelini alla chiesa San Lorenzo di Gatteo, sappiamo, dalla stessa fonte del 1575 prima citata, che nel 1555 conoscerà il futuro re Filippo II. Si evince che in quegli anni l'ingegnere militare riesce a partire con l'esercito spagnolo nel nuovo fronte di guerra tra Spagna e Francia: le Fiandre. Tra il 1556 ed il 1557 infatti un corposo esercito di spagnoli ed imperiali tenta l'ingresso a Parigi dalle colonie del nord. L'armata antifrancese era composta ancora una volta oltreché da spagnoli anche da molti italiani, uno su tutti il Duca di Savoia Emanuele Filiberto. Notizie sull'effettivo ruolo svolto dall'Antonelli nella campagna militare ci giungono in prima battuta dalle Epitomi. Antonelli cita due scontri, "Dorlano" che sarebbe Dourlens e San Quintino, divenuto celebre. Pare che per ciascuna l'ingegnere ebbe ruoli diversi, nella prima fu impegnato come aiutante al fianco di Jean de Ligne Duca di Aremberg. Questi era il Maestro di Campo dell'esercito guidato da Emanuele Filiberto, che assegnò tale ruolo all'Antonelli. Mentre durante la battaglia di San Quintino, Antonelli sembra ricoprire un ruolo più vicino al Capitano generale dell'artiglieria Juan Manrique de Lara, confermato nel proemio delle Epitomi sull'artiglieria. Seguì nel 1559 il trattato di pace di Cateau-Cambresis, e le truppe spagnole fecero il loro rientro in patria. Giovan Battista in quell'anno dovette arrivare in Spagna e si rifugiò alla Corte spagnola per scrivere le Epitomi ed 
attendere un nuovo incarico da Filippo II, che non tardò ad arrivare.

L'Antonelli partecipò ad una serie di guerre e battaglie storiche che fecero scuola presso gli strateghi militari poiché a quel tempo, trovarsi in un'area di conflitto, dove si sapevano esser confluiti le migliori teste sull'argomento, diventava quasi un titolo di credito, un attestato di nuove conoscenze.

\section{References}

Antonelli G. B., a cura di Sartor M., (2009). Epitomi delle fortificationi moderne. Forum Ed. Udine.

Biringuccio V., (1540) De la Pirotechnia. Ed. Venezia.

Lamberini D., (2007). Il Sanmarino: Giovan Battista Belluzzi, architetto militare e trattatista del Cinquecento. Leo S. Olschki Ed. Firenze.

Litta P., (1880) Famiglie celebri d'Italia. Ed. L. Bonadonna. Milano.

Useli G., (2013) Il Castello de Los Tres Reyes del Morro dell'Avana. Analisi del degrado e della consistenza statica del complesso, Tesi di laurea, Facoltà di Architettura della Università di Firenze. Archivio storico gentilizio dei conti Guidi di Bagno, Castello di Montebello, Torriana (RN). http://www.condottieridiventura.it/index.php/lettera-b/608-giovanni-francesco-da-bagno-i 
